\title{
Case Report of a 12-Year-0ld Girl with Imperforate Hymen-Review of the Literature on Incision Techniques and Further Treatment of Patients
}

\author{
Eftychia Kanioura, Evgenia Kalogridaki, Areti-Alexandra Methymaki, George Pantalos, \\ Ioannis Skondras*, Alexandros Passalides
}

$2^{\text {nd }}$ Surgical Department of Panagiotis and Aglaia Kyriakou Children’s Hospital, Athens, Greece

Email: *skondras@yahoo.gr

How to cite this paper: Kanioura, E., Kalogridaki, E., Methymaki, A.-A., Pantalos, G., Skondras, I. and Passalides, A. (2019) Case Report of a 12-Year-Old Girl with Imperforate Hymen-Review of the Literature on Incision Techniques and Further Treatment of Patients. Open Journal of Pediatrics, 9, 281-286.

https://doi.org/10.4236/ojped.2019.94026

Received: September 8, 2019

Accepted: October 27, 2019

Published: October 30, 2019

Copyright $\odot 2019$ by author(s) and Scientific Research Publishing Inc. This work is licensed under the Creative Commons Attribution International License (CC BY 4.0).

http://creativecommons.org/licenses/by/4.0/

\begin{abstract}
Background: Imperforate hymen (IH) is formed if no canal is created between the vaginal plate and the urogenital sinus. Treatment of IH is surgical. Different types of incisions and postoperative treatment are proposed. Case: A 12-year-old girl presented with a 3-day history of pain in the lower abdomen. Examination of the external genitalia disclosed a bulging hymen. Ultrasound examination of her lower abdomen revealed hematocolpos. A diagnosis of hematocolpos caused by IH was established and surgical treatment was decided. Conclusion: Treatment of choice for IH is undeniably surgical. Although different types of procedures have been proposed, the surgeon should choose the one that best suits the patient's needs without harming anatomical structures closely related to the hymen.
\end{abstract}

\section{Keywords}

Imperforate Hymen, Hematocolpos, Hymenectomy, Incision Techniques

\section{Introduction}

The hymen is a membrane located in the vaginal opening at the level of the introitus and is composed of urogenital sinus epithelial connective tissue [1] [2] [3] [4] [5]. During the final weeks of the embryologic development, a canal is created between the vaginal plate and the urogenital sinus [1] [2] [4] [6] [7] [8]. An imperforate hymen (IH) is a congenital disorder whereby the hymenal membrane covers the entire vaginal opening, thus causing genital outflow obstruction which leads to hematocolpos. The condition is mostly detected in ado- 
lescence when girls begin to menstruate [3] [4] [7] [8] [9] [10] [11]. The main presenting symptoms are cyclical abdominal pain and urinary retention in girls with a history of primary amenorrhea [1] [3]-[12]. The incidence of IH ranges from $1 / 1000$ to $1 / 10,000$ girls [1] [2] [3] [4] [5] [7] [8] [9] [11] [12]. Very rarely is it associated with familiar occurrence [1] [3] [8] [11] [12] or other female genital [2] [9] [11] [12] or urinary tract anomalies [3] [9]. The treatment of imperforate hymen is surgical and different types of incisions are proposed. The objective of this paper is to demonstrate a case of originally misdiagnosed $\mathrm{IH}$ and to discuss on the different treatments available.

\section{Case}

A 12-year-old girl presented to the emergency department of our hospital with a 3-day history of pain in the lower abdomen. No fever was reported. She had been admitted to the pediatric ward of our hospital 2 days earlier owing to urinary retention. During her hospitalization, a Foley catheter was inserted and urine flow was restored. Routine and culture urine samples were taken, the results of which were normal. The girl was discharged after 24 hours. No menstruation was noted. The rest of the girl's medical history was unremarkable.

Physical examination of the patient revealed pain in the lower abdomen. Giordano's sign was negative on both sides. Secondary sexual characteristics were found to be normal. Examination of the external genitalia disclosed a bulging hymen.

An ultrasound examination of her lower abdomen revealed hematocolpos (Figure 1). The rest of the internal genitalia showed normal development in accordance with her age. No further imaging examinations were performed. No pathological parameters resulted from blood and urine test.

A diagnosis of hematocolpos caused by IH was established and surgical treatment was decided. Surgery was performed under general anesthesia with the patient placed in the lithotomy position. The external genitalia area was sterilized and a Foley catheter was placed in the urethra so as to prevent it from being damaged (Figure 2). An X-shaped incision of the hymen was made and $600 \mathrm{ml}$ of dark fluid was slowly drained. No suction was used. Four stitches were applied at the rim of the remaining hymen to ensure adequate haemostasis. The Foley catheter was immediately removed following completion of the procedure. The girl urinated normally after surgery and no postoperative antibiotics or estrogen ointment was prescribed. Sterilization of the external genitalia with Betadine solution three times per day was recommended for the first 24 hours. She was discharged from hospital the following day. The follow-up period was uneventful. Her menstruation was normal and no reformation of the hymen has been reported to date. It was not possible to assess possible sex dysfunction since the patient was not yet sexually active.

\section{Discussion}

$\mathrm{IH}$ is the most common congenital obstructive anomaly of female genital tract 


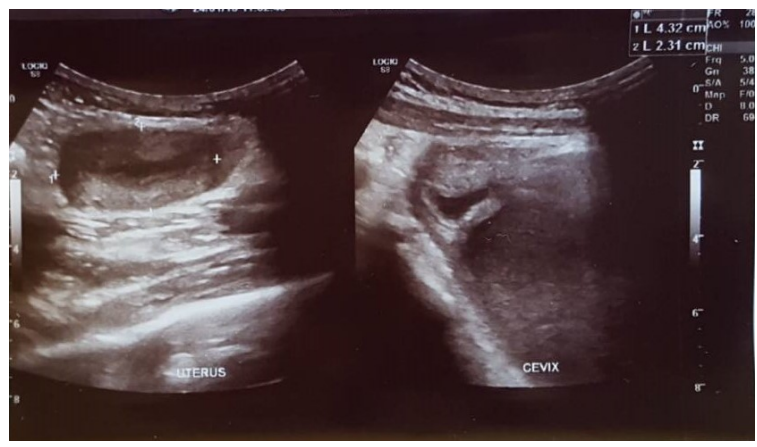

Figure 1. Ultrasound examination of the lower abdomen revealing hematocolpos.

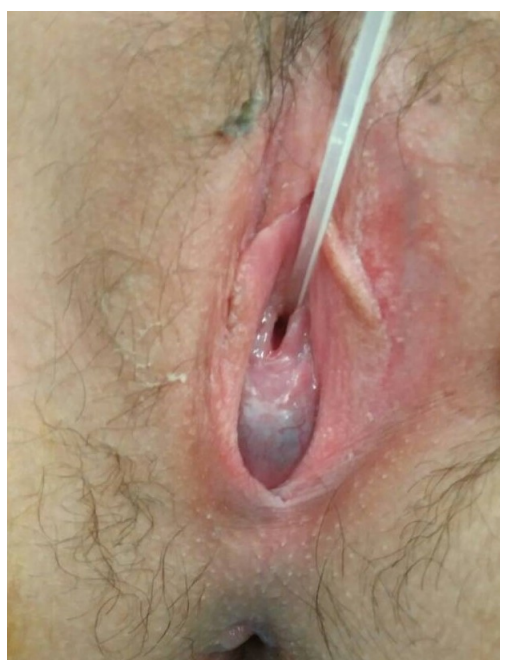

Figure 2. Patient placed in the lithotomy position for the surgery. A Foley catheter is placed in the urethra so as to prevent it from being damaged. A bulging hymen is obvious.

[2] [3] [4] [6] [12] [13]. It is usually detected in puberty due to hematocolpos [3] [4] [7] [8] [9] [10] [11] [14], although cases of infants have been reported with hydrocolpos secondary to IH [3] [4] [5] [9] [15]. Misdiagnosis of IH is not uncommon, as occurred in our case. Similar symptoms are common in low transverse vaginal septum [14], obstructed hemivagina and ipsilateral renal agenesis [16]. Delayed diagnosis of IH can cause several complications, the most frequent of which is endometriosis [2] [4] [6] [13], while less frequent complications include retrograde menstruation, pelvic adhesions, fallopian tube damage, infertility [16] [17]. Consequently, physicians should have a high index of suspicion when young girls present to them with symptoms that most commonly relate to $\mathrm{IH}$, i.e. cyclical lower abdominal pain and/or urinary outflow obstruction combined with the absence of menarche [17].

The treatment of IH is surgical and different types of incisions are proposed. In our case, as it was also reported in other articles [2] [16] an X-shaped incision was chosen combined with the insertion of a Foley catheter in the urethra so as to stent it. This method protects the integrity of the urethra [2]. Despite its supe- 
riority in maintaining the urethra intact, the $\mathrm{X}$-shaped incision is not suggested in the vast majority of reports published in the literature. A cross-shaped incision of the hymen, with or without the use of a Foley catheter to stent the urethra, seems to be the incision of choice [1] [3] [7] [8] [11]. Other types of incision include: a star-incision so as to protect the Bartholin's glands [9], vertical midline incision $(<1 \mathrm{~cm})$ [12], annular incision with [4] or without [6] [10] [13] placement of a Foley catheter in the opening of the hymen and an elliptical incision [16]. The annular incision is claimed to have the least effect on the hymen's architecture and thus appears to be the preferred choice among cultures and religions where a girl's virginity is of great importance [4] [6].

Apart from the type of incision, a differentiation has been noticed in the use or not of stitches to prevent the reformation of the hymen. In our case, stitches were used as a means of haemostasis and no reformation of the hymen was reported. Not only in cases where stitches were applied [2] [3] [8] [12] [13] but also in those where they were not used [1] [6], no reformation of the hymen was detected. Only one case of reformation after four repeated surgeries is reported [4].

The surgical instrument used to perform the incision in our case was a No. 11 lancet, as used for most cases in the literature. Only two different techniques have been reported: one demonstrates the use of unipolar electroautery [6] and the other the use of $\mathrm{CO}_{2}$ laser [10]. Finally, controversy appears to surround postoperative. Some authors report the use of antibiotics [2] [9] and estrogen ointment [9], while others choose to retain the Foley catheter in the incision for two weeks [9]. In our case, none of the above measures were taken. We chose to advise application of Betadine solution to the area, three times a day for the first 24 hours after the procedure. With the exception of one case [4], all patients had an uneventful recovery and follow-up period, as did our patient.

\section{Conclusion}

In conclusion, IH is a condition whose diagnosis often mandates a high index of suspicion on behalf of the physician. If left untreated, it could result in serious health problems for the patient. The treatment of choice is undeniably surgical. Although different types of procedures have been proposed, the surgeon should choose the procedure that best suits the patient's needs without harming anatomical structures closely related to the hymen.

\section{Acknowledgements}

The authors have no acknowledgements to make.

\section{Authorship}

All authors meet the ICMJE criteria for authorship.

\section{Funding Sources}

This research did not receive any specific grant from funding agencies in the 
public, commercial, or not-for-profit sectors.

\section{Ethics}

The procedures followed were in accordance with the ethical standards of the Helsinki Declaration of 1975, as revised in 1983.

A written consent form was given by the patient's parents in which their agreement to publish this report was stated.

\section{Conflicts of Interest}

The authors declare no conflicts of interest regarding the publication of this paper.

\section{References}

[1] Papeš, D., Arslani, N., Rajković, Z., Altarac, S. and Kopjar, M. (2011) An Unusual Cause of Anuria and Hydronephrosis in a 12-Year-Old Girl. Renal Failure, 33, 540-543. https://doi.org/10.3109/0886022X.2011.569106

[2] Mwenda, A.S. (2013) Imperforate Hymen-A Rare Cause of Acute Abdominal Pain and Tenesmus: Case Report and Review of the Literature. The Pan African Medical Journal, 21, 58. https://doi.org/10.11604/pamj.2013.15.28.2251

[3] Eskandar, O. and Wheble, A. (2004) Imperforate Hymen in Two Sisters Associated with Renal Angiomyolipoma and Duplex Kidney. Journal of Obstetrics and Gynaecology, 24, 922-993. https://doi.org/10.1080/01443610400019054

[4] Ossman, A.M.E., El-Masry, Y.I., EL-Namoury, M.M. and Sarsik, S.M. (2016) Spontaneous Reformation of Imperforate Hymen after Repeated Hymenectomy. Journal of Pediatric and Adolescent Gynecology, 29, 63-65. https://doi.org/10.1016/j.jpag.2016.02.001

[5] Liang, C.C., Chang, S.D. and Soong, Y.K. (2003) Long-Term Follow-up of Women Who Underwent Surgical Correction for Imperforate Hymen. Archives of Gynecology and Obstetrics, 269, 5-8. https://doi.org/10.1007/s00404-002-0423-3

[6] Cetin, C., Soysal, C. and Khatib, G. (2016) Annular Hymenotomy for Imperforate Hymen. Journal of Obstetrics and Gynaecology Research, 42, 1013-1015. https://doi.org/10.1111/jog.13010

[7] Mou, J.W., Tang, P.M., Chan, K.W., Tam, Y.H and Lee, K.H. (2009) Imperforate Hymen: Cause of Lower Abdominal Pain in Teenage Girls. Singapore Medical Journal, 50, 378-379.

[8] Dane, C., Dane, B., Erginbas, M. and Ceti, A. (2007) Imperforate Hymen-A Rare Cause of Abdominal Pain: Two Cases and Review of the Literature. Journal of Pediatric and Adolescent Gynecology, 20, 245-247. https://doi.org/10.1016/j.jpag.2006.12.003

[9] Ali, A., Cetin, C., Nedim, C., Kazim, G and Cemalettin, A. (2003) Treatment of Imperforate Hymen by Application of Foley Catheter. The European Journal of Obstetrics \& Gynecology and Reproductive Biology, 106, 72-75. https://doi.org/10.1016/S0301-2115(02)00214-2

[10] Frega, A., Verrone, A. and Schimberni, M. (2015) Feasibility of Office $\mathrm{CO}_{2}$ Laser Surgery in Patients Affected by Benign Pathologies and Congenital Malformations of Female Lower Genital Tract. European Review for Medical and Pharmacological Sciences, 19, 2528-2536. 
[11] Chang, J.W., Yang, L.Y., Wang, J.-K., Tiu, C.-M. and Wang, H.H. (2007) Acute Urinary Retention as the Presentation of Imperforate Hymen. Journal of the Chinese Medical Association, 70, 559-561.

https://doi.org/10.1016/S1726-4901(08)70061-3

[12] Basaran, M., Usal, D. and Aydemir, C. (2009) Hymen Sparing Surgery for Imperforate Hymen: Case Reports and Review of Literature. Journal of Pediatric and Adolescent Gynecology, 22, 61-64. https://doi.org/10.1016/j.jpag.2008.03.009

[13] Laghzaoui, O. (2016) Congenital Imperforate Hymen. BMJ Case Reports, 2016, bcr2016215124. https://doi.org/10.1136/bcr-2016-215124

[14] Bekaert, T. and Ramboer, K. (2018) Hematometrocolpos Due to an Imperforate Hymen. Journal of the Belgian Society of Radiology, 102, 1-2. https://doi.org/10.5334/jbr-btr.1413

[15] Peleg, D. and Shinwel, E.S. (2019) Newborn Imperforate Hymen Resulting in Hydronephrosis. The Journal of Pediatrics, 207, 258. https://doi.org/10.1016/j.jpeds.2018.12.051

[16] Egbe, T.O., Kobenge, F.M. and Wankie, E.M. (2019) Virginity-Sparing Management of Hematocolpos with Imperforate Hymen: Case Report and Literature Review. SAGE Open Medical Case Reports, 7, 1-7. https://doi.org/10.1177/2050313X19846765

[17] Kotter, H.C., Weingrow, D. and Canders, C. (2017) Hematometrocolpos in a Pubescent Girl with Abdominal Pain. Clinical Practice and Cases in Emergency Medicine, 1, 218-220. https://doi.org/10.5811/cpcem.2017.3.33369 